\title{
Efeito da Berinjela sobre os Lípides Plasmáticos, a Peroxidação Lipídica e a Reversão da Disfunção Endotelial na Hipercolesterolemia Experimental
}

\author{
Paulo A fonso Ribeiro Jorge, Lidia C. N eyra, Regina M. O saki, Eros de Almeida, N eura Bragagnolo \\ Campinas, SP
}

Objetivo - Estudar o efeito do suco da berinjela sobre os lípides plasmáticos, o colesterol tecidual, a peroxidação lipídica das LDL nativas, oxidadas e da parede arterial e o relaxamento dependente do endotélio, em coelhos hipercolesterolêmicos.

Métodos - Coelhos foram separados em grupos controle $(G C)$, hipercolesterolêmico $(G H)$ e berinjela $(G B)$, $(n=10)$. Os animais do GC foram alimentados com ração normal, o GH e o GB com ração acrescentada de colesterol $(0,5 \%)$ e gordura de babaçu (10\%) durante 30 dias. Ao GB acrescentou-se suco de berinjela, nos últimos 15 dias do experimento. Os lípides plasmáticos foram medidos através de kits enzimáticos, a peroxidação lipídica pela dosagem do malondialdeído (MDA) e o relaxamento dependente do endotélio, por curvas de concentração efeito pela acetilcolina e nitroprussiato.

Resultados - O peso dos animais foi menor no $\mathrm{GB}$ em relação ao $G C$ e $G H(p<0,05)$. O colesterol total plasmático, as LDL e os triglicérides que se elevaram no $G H$, reduziram-se em 19\%, 29\% e 38\%, respectivamente no GB ( $p<0,05)$. O colesterol tecidual foi menor no GB (50\%) em relação ao $G H(p<0,05)$. Nos animais do GB ocorreu redução significante do teor de MDA nas LDL nativas $(56 \%)$ e oxidadas $(22 \%)$, assim como na parede arterial $(30 \%)(p<0,05)$. O relaxamento máximo dependente do endotélio, , elevou-se significantemente em $28 \%$ no $\mathrm{GB}$ em relação ao $\mathrm{GH}(\mathrm{p}<0,05)$.

Conclusão - O suco de berinjela administrado a coelhos hipercolesterolêmicos reduziu significantemente o peso corpóreo, o colesterol total, as LDL plasmáticas, os triglicérides, o colesterol tecidual, a peroxidação lipídica das LDL nativas, oxidadas e da parede arterial, assim como aumentou o relaxamento dependente do endotélio.

Palavras-chave: berinjela (solanum melongena), LDL, lípides plasmáticos, colesterol
Effect of Eggplant on Plasma Lipid Levels, Lipidic Peroxidation and Reversion of Endothelial Dysfunction in Experimental Hypercholesterolemia

Purpose - To study the effect of egg plant on endothelium-dependent relaxation, and plasma lipids in hypercholesterolemic rabbits, and to assess influence of this plant on the malondialdehyde (MDA) content of LDL particles and the arterial wall.

Methods - Thirteen male rabbits were randomly as signed to control $(C)$, hypercholesterolemic $(H)$ and egg plant $(E)$ treated groups $(n=10$ each $)$. The H and E rabbits were fed a diet supplemented with cholesterol (0.5\%) and coconut oil (10\%) for 4 weeks. In addition, group E received $10 \mathrm{~mL}$ of the fruit juice/day during the last 2 weeks.The animals were killed and the aorta removed to measure MDA content and the endothelium dependent relaxation responses. Total plasma cholesterol, VLDL, LDL, $H D L$ and triglyceride levels were determined using commercial kits. MDA was quantified in native and oxidized $L D L$ and in the arterial wall.

Results - After 4 weeks, the E group rabbits had a significantly lower weight, plasma cholesterol, LDL, triglyceride and aortic cholesterol contenthan group $H(p<0.05)$. The MDA content that was significantly increased in the LDL particles and in the arterial wall of $H$ rabbits was reduced in the E group ( $p<0.05)$. Endothelium-dependent relaxation were significantly higher in the E group compared H group rabbits ( $p<0.05)$.

Conclusion - In hypercholesterolemic rabbits egg plant juice significantly reduced weight, plasma cholesterol levels, aortic cholesterol content and the MDA concentrations in native-oxidized LDL and in the arterial wall and increased the endothelium-dependent relaxations.

Kew-words: eggplant, cholesterol, oxidized LDL

Arq Bras Cardiol, volume 70 (n 2), 87-91, 1998

Faculdades de Ciências Médicas - UNICAMP, Campinas. Trabalho realizado com bolsa da FAEP.

Correspondência: Paulo Afonso Ribeiro Jorge - Rua Guilherme da Silva, 397/31 - 13025-070 - Campinas, SP

Recebido para publicação em 1/9/97

Aceito em 3/12/97
A hipercolesterolemia e sua relação com a doença coronária aterosclerótica tem sido demonstrada em muitos ensaios clínicos ${ }^{1,2}$. De outro lado, a redução do número de eventos e de mortalidade ou doença coronária, a interrupção ou mesmo a regressão da doença aterosclerótica atra- 
vés de drogas redutoras do colesterol plasmático, também tem sido referida por inúmeros estudos ${ }^{3-7}$. Os mecanismos envolvidos na redução dos eventos e na mortalidade por doença coronária, quando ocorre diminuição do colesterol plasmático, parecem estar relacionados à reversão da disfunção endotelial e estabilização da placa de aterosclerose, uma vez que não se observa aumento significante do diâmetro vascular ao nível da placa de aterosclerose ${ }^{4}$.

Mais recentemente, tem-se verificado que as substâncias anti-oxidantes são capazes de reverter a disfunção endotelial provocada pela hipercolesterolemia ${ }^{8,9}$ e também reduzir o numero de eventos coronários ${ }^{10}$, embora sua utilização, na prática médica, careça, ainda, de informação mais conclusivas.

Diante destas evidências, a redução do colesterol plasmático e a proteção endotelial colocam-se como importantes providências para o controle da doença aterosclerótica.

Considerando o elevado custo dos medicamentos redutores do colesterol plasmático e a perspectiva de seu uso prolongado, os pacientes têm recorrido a tratamentos alternativos para o controle da hipercolesterolemia. Estes tratamentos têm sido utilizados de forma empírica pela população, carecendo de uma metodologia de estudo que permita conclusões mais confiáveis. Em nosso meio, tem sido usada a berinjela, sob a forma de suco do fruto, ingerido duas ou três vezes ao dia. A ampla utilização da berinjela pela população, de um modo geral, e a importância do melhor conhecimento de seu efeito sobre a hipercolesterolemia e a proteção endotelial, representou o fundamento desta proposta de estudo, que visa verificar o efeito do suco de berinjela sobre o peso corpóreo, os lípides plasmáticos (colesterol total (CT), triglicérides (TG), LDL-colesterol e HDLcolesterol), o colesterol da parede arterial, a peroxidação das HDL-plasmáticas e da parede arterial e o relaxamento dependente do endotélio, em coelhos hipercolesterolêmicos.

\section{Métodos}

Foram utilizados coelhos da raça Nova Zelândia, machos, com peso médio de $2.530 \pm 0,10 \mathrm{~g}$ e idade média de oito semanas, separados em três grupos: grupo controle (GC) $(n=10)$; grupo hipercolesterolemia $(\mathrm{GC})(\mathrm{n}=10)$ e grupo berinjela (GB) $(n=10)$.

Os coelhos do GC foram alimentados com ração da marca Criacoelhos (Mogiana Alimentos S/A), na quantidade de $100 \mathrm{~g}$ ao dia e com a seguinte composição em g/100: proteínas, 20,00; carboidratos, 45,00; fibras, 16,00; gordura, 5,00; cinzas, 14,00. Os animais dos GH e GB receberam a mesma dieta do GC, enriquecida com colesterol $(0,5 \%)$ e gordura de babaçu (10\%), durante 30 dias. Aos coelhos do GB foram administrados $10 \mathrm{~mL} /$ dia de suco de berinjela, durante os últimos 15 dias do experimento, através de gavagem.

O preparo do suco de berinjela constou de $100 \mathrm{~g}$ de berinjela e $70 \mathrm{~mL}$ de água, batidos em liqüidificador durante 5 min e foi estudado através de análise físico-química de acordo com os métodos oficiais da associação de análises químicas ${ }^{11}$, apresentando as seguintes características: em g/100 - proteínas 0,74 ; carboidratos 2,72; fibras 0,40; lípides totais 0,34 ; cinzas 0,26 ; calorias, 17 calorias.

Não encontramos na literatura referência sobre a dose a ser administrada. Estabelecemos, de forma empírica, a dose de $10 \mathrm{~mL}$ do suco de berinjela, que corresponde a $0,10 \mathrm{~g}$ de proteínas. Esta dose foi bem aceita pelos animais em projeto piloto, sem qualquer reação adversa.

A dosagem do CT, LDL, HDL e TG foi realizada em amostras de sangue, obtidas por punção cardíaca, utilizando-se kits enzimáticos e espectofotômetro da marca Bechman 700.

No final do experimento os animais foram sacrificados, sendo retirada a aorta toráxica. O colesterol em anéis de aorta foi medido de acordo com o método de Naito e David ${ }^{12}$. Os espécimes foram secos e homogeneizados a $4^{\circ} \mathrm{C} \mathrm{em} 5 \mathrm{~mL}$ de tampão TrisHCL, pH 7,4 acrescido de 0,01 NaNO3. Os lípides totais foram extraídos e homogeneizados em 10 vol de clorofórmio-metanol OCT foi medido por ensaio enzimático.

Quanto à dosagem da peroxidação lipídica da parede arterial, um segmento da aorta torácica foi homogeneizado com ácido tricloroacético (1g de tecido + 10 vol TCA 20\%). Após centrifugação, foi acrescentado um volume de ácido tiobarbitúrico $0,67 \%$ e a mistura aquecida a $100^{\circ} \mathrm{C}$ por $20 \mathrm{~min}$. A concentração de malondialdeído (MDA) foi calculada pela absorbância de 532nm, utilizando-se um coeficiente de extinção de $1,49 \times 10^{-5}$ expresso em moL/mg de tecido x $10^{-7}$.

Com relação à dosagem da peroxidação lipídica das LDL, amostras de sangue foram coletadas em tubos plásticos com EDTA a pH 7,4 e centrifugadas a 3.000rpm por $15 \mathrm{~min}$ a $10^{\circ} \mathrm{C}$. Este procedimento remove todos os resíduos celulares e é obtido um plasma completamente claro. O método utiliza o procedimento de Havel e col ${ }^{13}$ que consiste em dois passos seqüenciais de ultracentrifugação por 48h. Inicialmente, $4 \mathrm{~mL}$ de plasma é centrifugado a $40.000 \mathrm{rpm}$ por $18 \mathrm{~h}$ (ultracentrífuga Beckman, mod L-8) para retirar os quilomícrons e VLDL. Com uma pipeta Pasteur são retiradas es-

\begin{tabular}{|c|c|c|c|}
\hline & $\mathrm{GH}$ & GB & CG \\
\hline Coelhos (n) & 10 & 10 & 10 \\
\hline Peso (kg) & $2,98 \pm 0,098^{\mathrm{a}}$ & $2,22 \pm 0,14^{\mathrm{b}}$ & $2,95 \pm 0,08^{\mathrm{a}}$ \\
\hline $\mathrm{CT}(\mathrm{mg} / \mathrm{dL})$ & $1360,51 \pm 159,22^{\mathrm{a}}$ & $1097,70 \pm 315,53^{b}$ & $59,60 \pm 5,39^{c}$ \\
\hline VLDL (mg/dL) & $410,53 \pm 42,50^{\mathrm{a}}$ & $435,89 \pm 130,07^{\mathrm{a}}$ & $8,92 \pm 2,22^{\mathrm{b}}$ \\
\hline $\mathrm{LDL}(\mathrm{mg} / \mathrm{dL})$ & $841,33 \pm 240,62^{\mathrm{a}}$ & $595,87 \pm 207,06^{c}$ & $16,91 \pm 2,40^{\mathrm{c}}$ \\
\hline $\operatorname{HDL}(\mathrm{mg} / \mathrm{dL})$ & $49,19 \pm 4,63^{\mathrm{a}}$ & $54,76 \pm 15,32^{\mathrm{a}}$ & $32,85 \pm 5,15^{\mathrm{b}}$ \\
\hline $\mathrm{TG}(\mathrm{mg} / \mathrm{dL})$ & $326,54 \pm 78,61^{\mathrm{a}}$ & $203,41 \pm 36,26^{\mathrm{b}}$ & $119,00 \pm 35,26^{\mathrm{c}}$ \\
\hline MDA-LDL oxidada & $57,23 \pm 5,26^{\mathrm{a}}$ & $44,67 \pm 7,42^{\mathrm{b}}$ & $31,21 \pm 5,06^{\mathrm{c}}$ \\
\hline MDA-LDL nativa & $3,31 \pm 0,65^{\mathrm{a}}$ & $1,45 \pm 0,92^{\mathrm{b}}$ & $1,49 \pm 0,46^{b}$ \\
\hline MDA-aorta & $9,19 \pm 0,94^{\mathrm{a}}$ & $6,43 \pm 0,43^{b}$ & $5,20 \pm 0,61^{\mathrm{c}}$ \\
\hline Col. tecidual & $30,71 \pm 2,06^{\mathrm{a}}$ & $15,35 \pm 2,58^{\mathrm{c}}$ & $21,44 \pm 4,37^{\mathrm{b}}$ \\
\hline Tensão & $1,37 \pm 0,28$ & $1,60 \pm 0,12$ & $1,50 \pm 0,39$ \\
\hline Relaxamento máx. & $49,37 \pm 5,55^{\mathrm{c}}$ & $63,34 \pm 6,05^{\mathrm{b}}$ & $92,11 \pm 6,05^{\mathrm{a}}$ \\
\hline \multicolumn{4}{|c|}{$\begin{array}{l}\text { CT- colesterol total; TG- triglicérides; MDA-LDL native a oxidada- nanomoles } \\
\text { de malondialdeído/mg de proteína de LDL; MDA-aorta- nanomoles de } \\
\text { malondialdeído/mg de tecido, col. tecidual- colesterol da aorta }(\mathrm{mg} / \mathrm{mg}) \text {; relaxa- } \\
\text { mento max- relaxamento máximo dependente do endotélio. Letras distintas indi- } \\
\text { cam diferença significantes entre grupos }(\mathrm{p}<0,05) \text {. Teste: Kruskall-Wallis. }\end{array}$} \\
\hline
\end{tabular}


tas lipoproteínas e a densidade ajustada para $1,063 \mathrm{~g} / \mathrm{mL}$ com KBr sólido. Outra centrifugação a 40.000rpm durante 20h, permitirá isolar as LDL, que serão coletadas com uma pipeta de ponta fina, para sua caracterização e oxidação.

O colesterol das LDL isoladas foi determinado através de kit enzimático da Boehringer Manheim, Germany. A proteína foi quantificada pelo método de Lowry e $\mathrm{col}^{14}$. A oxidação da LDL foi realizada mediante incubação de $100 \mu \mathrm{g}$ de proteína/mL em tampão fosfato-salino $1 \mathrm{mH}, \mathrm{pH} 7,4$ em presença de sulfato de cobre $(5 \mathrm{mM})$ a $37^{\circ} \mathrm{C}$, por $24 \mathrm{~h}$. A dosagem de TBARS consistiu em colocar em um tubo de ensaio, $150 \mu \mathrm{g}$ de proteína de LDL, completando para um volume de $500 \mu \mathrm{g}$, na presença de $1 \mathrm{~mL}$ de ácido tiobarbitúrico $0,67 \%$ e $0,3 \mathrm{~mL}$ de ácido tricloroacético a $50 \%$. Após aquecimento a $100^{\circ} \mathrm{C}$ por $30 \mathrm{~min}$, o teor das substâncias reativas com ácido tiobarbitúrico foi determinado pelo método de Bueg e Aust ${ }^{15}$, com leitura a 532nm de absorbância.

Anéis da aorta de aproximadamente $5 \mathrm{~mm}$ foram obtidos tomando-se especial cuidado para manter intacto o endotélio. O endotélio foi mecanicamente removido em igual número de anéis de aorta, atritando-se à superfície endotelial do vaso com uma pequena cureta. Os anéis foram suspensos em uma solução de Krebs-Henseleit a $37^{\circ} \mathrm{e} \mathrm{pH}$ 7,4 em uma cuba de $10 \mathrm{~mL}$. A solução foi continuadamente aerada com mistura de carbogênio contendo $95 \%$ de oxigênio e 5\% de gás corbônico. Os anéis de aorta foram montados em dois ganchos de metal ligados a um suporte da cuba e a um transdutor de força. Os espécimes foram deixados em equilíbrio por $60 \mathrm{~min}$, com troca do Krebs a cada $20 \mathrm{~min}$. Em seguida foram distendidos a uma tensão basal de $1 \mathrm{~g}$. Todos os anéis de aorta, com e sem endotélio, foram contraídos com NE $\left(10^{-7} \mathrm{M}\right)$. Depois que a contração estabilizou-se, ACh foi adicionada de forma cumulativa $\left(10^{-8} \mathrm{a} 10^{-5} \mathrm{M}\right)$. $\mathrm{O}$ mesmo procedimento foi realizado para os anéis de aorta nos quais o endotélio foi removido.

As curvas que expressam o relaxamento dependente do endotélio foram comparadas por análise de co-variância ${ }^{16}$. Os valores de peso corpóreo, do CT, LDL, HDL, VLDL, TG, colesterol residual e MDA nos diferentes grupos de
Efeito da berinjela na hipercolesterolemia experimental

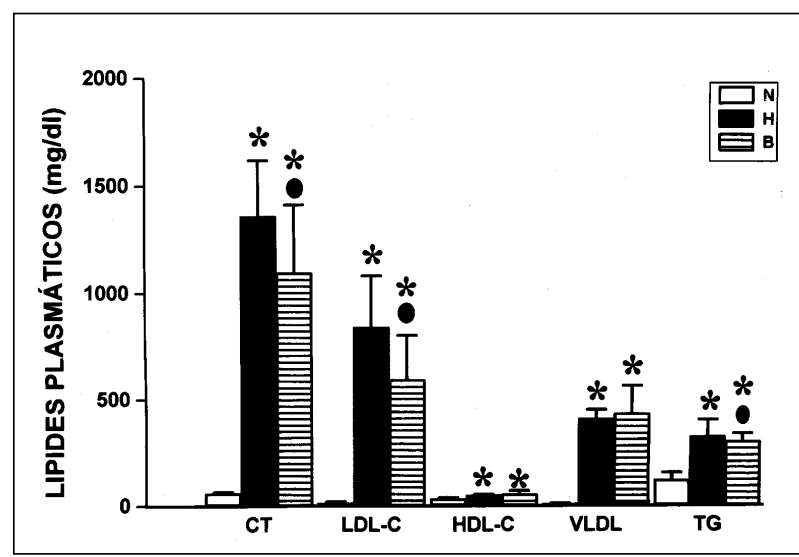

Fig. 1 - Lípides plasmáticos. Valores expressos em mg/dL representando média e desvio padrão. CT- colesterol total; LDL-C- lipoproteína de baixa densidade; HDL-Clipoproteína de alta densidade; VLDL-C- lipoproteína de muito baixa densidade; TG- triglicérides; N- grupo controle; H- grupo hipercolesterolemia; B- grupo berinjela. * $\mathrm{p}<0,05$ do controle $(\mathrm{N}) ;^{\circ} \mathrm{p}<0,05$ entre GH e GB.

animais foram examinados por teste estatístico não paramétrico de Kruskal-Wallis e Man-Withney ${ }^{17}, \mathrm{p}<0,05$ foi considerado significante.

Acetilcolina, nitroprussiato de sódio, norepinefrina e as substâncias da solução de Krebs-Henseileit foram obtidos de Sigma Co. (St Louis, MO). O colesterol empregado na dieta da Amresco (Solon, Ohio) e a gordura de coco de babaçu da Refino de Óleos Brasil Ltda.

\section{Resultados}

Os valores do peso corpóreo, do perfil lipídico, do MDA das LDL nativas, oxidadas e da parede da aorta, do colesterol tecidual e do relaxamento máximo dependente do endotélio estão expressos na tabela I.

Verificou-se que o peso corpóreo dos animais hipercolesterolêmicos tratados com berinjela, foi significantemente menor que dos animais dos $\mathrm{GCe} \mathrm{GH}(\mathrm{p}<0,05)$. Os valores do CT plasmático, LDL, VLDL e os TG aumentaram significantemente no GH quando comparado com o GC. Os

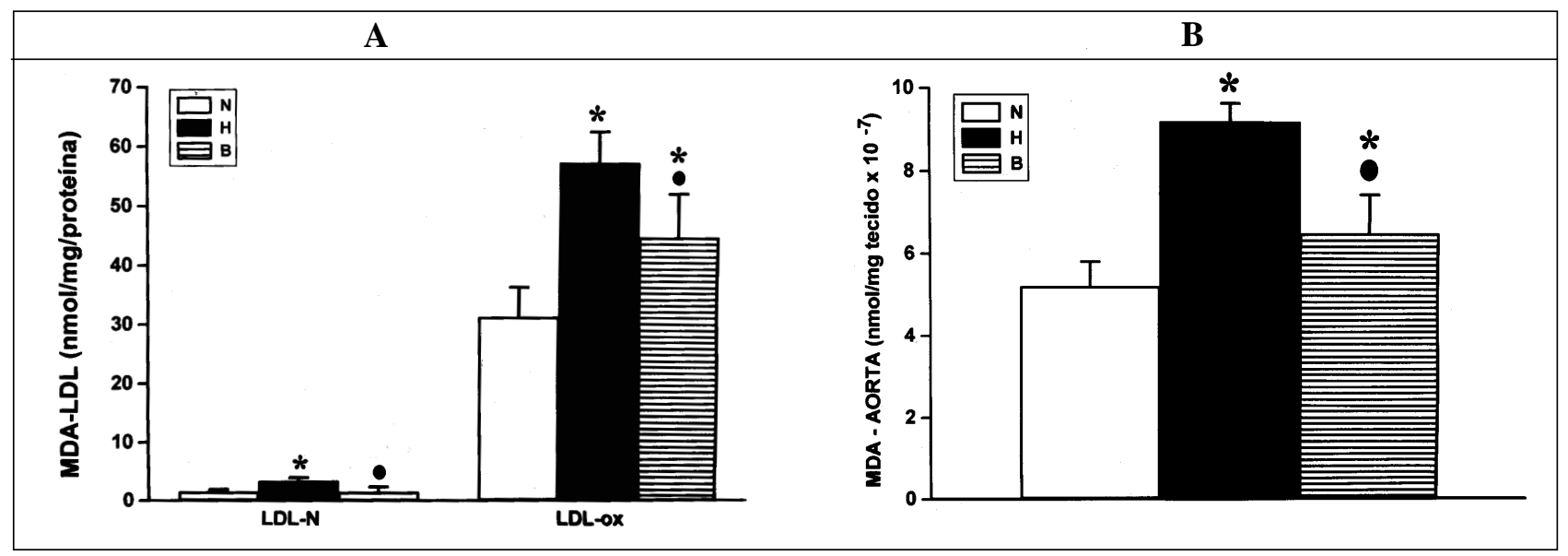

Fig. 2 - Concentração média e desvio padrão de malondialdeído (MDA) das LDL-nativas, oxidadas (A) e da parede arterial (B). Os valores estão expressos em nmoL/mg de proteína e nmoL/mg tecido. LDL-N- LDL nativa; LDL-ox- LDL oxidada; $\mathrm{N}$ - grupo controle; $\mathrm{H}$ - grupo hipercolesterolemia; $\mathrm{B}$ - grupo berinjela; ${ }^{*} \mathrm{p}<0,05$ em relação ao controle $(\mathrm{N}) ;{ }^{\circ} \mathrm{p}<0,05$ entre GHe GB 


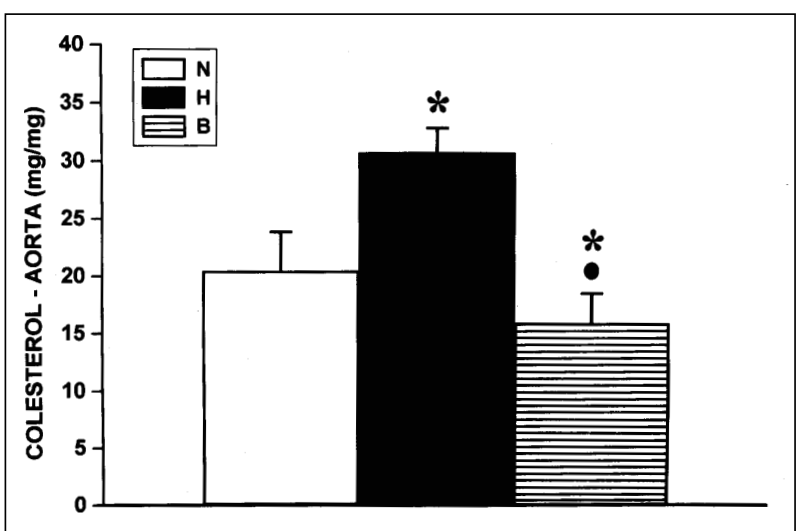

Fig. 3 - Valores médios e desvio padrão do colesterol da aorta. Os valores estão expressos em mg/mg; $\mathrm{N}$ - grupo controle; $\mathrm{H}$ - grupo hipercolesterolemia; $\mathrm{B}$ - grupo berinjela; * $\mathrm{p}<0,05$ para o controle $(\mathrm{N}){ }^{\circ} \mathrm{p}<0,05$ entre GH e GB.

valores do colesterol total, LDL e dos TG reduziram-se significantemente nos animais do GB, quando comparados como GH $(\mathrm{p}<0,05)$. Não houve diferença significante para os valores das VLDLe HDLentre os grupos $\mathrm{HeB}(\mathrm{p}<0,05)$ (fig. 1).

Os valores do MDA das LDL-nativas e oxidadas aumentaram significantemente nos animais do $\mathrm{GH}$ em relação ao GC $(\mathrm{p}<0,05)$ e reduziram-se significantemente nos animais do GB, quando comparados com o GH (p<0,05) (fig. 2). $\mathrm{O}$ teor de MDA da parede da aorta elevou-se no $\mathrm{GH}$ $(\mathrm{p}<0,05)$, quando comparado com o GC, e reduziu-se em relação ao GB (p<0,05) (fig. 3). O colesterol da aorta que elevou-se significantemente no $\mathrm{GH}$, quando comparado ao GC ( $p<0,05)$, reduziu-se em relação ao GB (p<0,05) (fig. 4). A comparação das curvas de concentração-efeito com acetilcolina, entre os animais do GHe o GC, revelou redução do percentual de relaxamento dependente do endotélio no $\mathrm{GH}(\mathrm{p}<0,05)$. Entretanto, observou-se aumento do percentual de relaxamento dependente do endotélio para o GB quando comparado com o GH (p<0,05). Não houve diferença entre os grupos $\mathrm{C}, \mathrm{H}$ e $\mathrm{B}$ quando as curvas de concentração-efeito foram obtidas com o nitroprussiato. A tensão de contração com noradrenalina não foi diferente nos três gru-

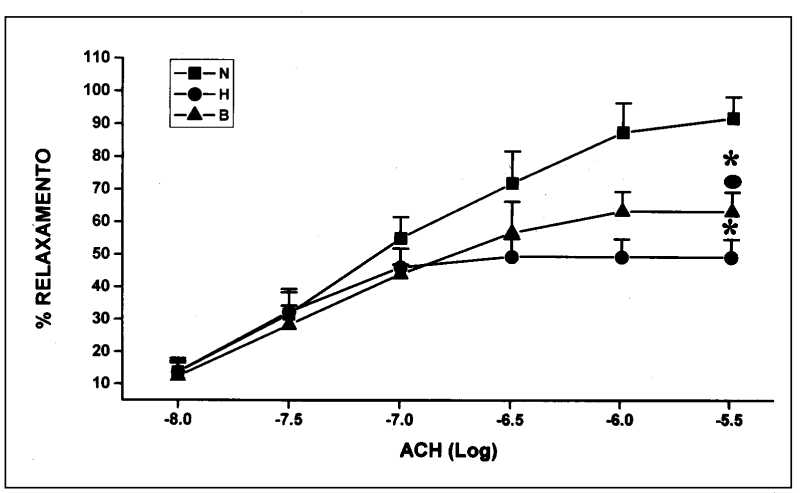

Fig. 4 - Curvas do relaxamento-dependente do endotélio em resposta à acetilcolina (ACh) após contração com norepinefrina (NE). A abcissa refere-se à concentração $(\log \mathrm{M})$ de acetilcolina e a ordenada ao relaxamento expresso como percentual da contração à $\mathrm{NE}\left(10^{-7} \mathrm{M}\right) ; \mathrm{N}$ - grupo controle; $\mathrm{H}$ - grupo hipercolesterolemia; $\mathrm{B}$ - grupo berinjela; ${ }^{*} \mathrm{p}<0,05$ em relação ao controle $(\mathrm{N}){ }^{\circ} \mathrm{p}<0,05$ entre GH e GB. pos estudados. Apesar de não terem sido observadas diferenças nas quantidades de alimento consumidas pelos três grupos durante o período de estudo, verificou-se que o peso dos animais hipercolesterolêmicos tratados com berinjela foi menor.

\section{Discussão}

A administração da dieta enriquecida com colesterol a $0,5 \%$ e gordura de coco de babaçu a $10 \%$, a coelhos da raça Nova Zelândia, durante 30 dias, mostrou acentuada elevação do CT plasmático (2183\%), das LDL (4875\%), das VLDL (4512\%), das HDL (52\%) e dos TG (174\%), quando comparado aos animais que receberam ração normal. Os valores do MDA das LDL nativas e oxidadas in vivo através da exposição ao Cu++, também se elevaram a valores de $122 \%$ e $83 \%$, respectivamente. $\mathrm{O}$ teor de MDA da parede da aorta elevou-se em $77 \%$. O colesterol da parede da aorta aumentou $43 \%$. Em conseqüência, ocorreu redução do percentual de relaxamento das curvas de concentração efeito com acetilcolina, sendo que o relaxamento máximo decresceu em $46 \%$. Estes achados estão em acordo com experimentos relatados na literatura e reafirmam que a hipercolesterolemia acentua a peroxidação lipídica e compromete a função endotelial ${ }^{18-20}$.

Nos animais hipercolesterolêmicos tratados com suco de berinjela por 15 dias, observou-se redução significante do CT plasmático em 19\%, quando comparado com o GH. O colesterol VLDL e o HDL não apresentaram modificações significantes. O colesterol LDL reduziu-se em $29 \%$ e os TG em $38 \%$. O MDA das LDL-oxidadas reduziu-se em $22 \%$ e o das LDL nativas em 56\%. O colesterol tecidual reduziu-se significantemente em 50\%, o MDA tecidual em $30 \%$ e o aumento do relaxamento máximo dependente do endotélio foi de $28 \%$.

Não há referência na literatura pesquisada, sobre o efeito do suco de berinjela nos parâmetros analisados neste estudo, em animais de experimentação. Mitschek GH ${ }^{21}$ estudou o efeito da berinjela sobre a aterosclerose experimental e concluiu, através de estudo histológico, que as alterações da parede arterial, com alargamento do espaço subendotelial, edema e presença de gotículas de lípides observados em coelhos hipercolesterolêmicos apareciam apenas como discreto edema no grupo tratado com berinjela. Em nota prévia, Cruz e col ${ }^{22}$ relataram o efeito da berinjela crua, liqüefeita com suco de laranja, administrada a pacientes hipertensos e descreveram uma redução do CT plasmático, das frações LDLe VLDL sem alteração dos valores da HDL.

A berinjela é uma planta da família das solanáceas, rica em vitaminas A, B1, riboflavina, niacina e ácido ascórbico ${ }^{23}$. Para explicar a redução do colesterol plasmático, Kritchvsky e $\operatorname{col}^{24}$ sugerem que ocorre inibição na absorção do colesterol, por ligação de algum componente da berinjela com sais biliares essenciais na absorção intestinal do colesterol. A presença da niacina, também, pode influir na redução do colesterol plasmático. A diminuição da peroxidação lipídica, observada nesse estudo, sugere o envolvimento de outras substâncias, cujos mecanismos de ação não constituem 
objeto deste trabalho. A redução do colesterol tecidual deve estar relacionada não só a diminuição do colesterol plasmático, mas também à redução da peroxidação lipídica das LDL nativa, oxidadas e da parede $\operatorname{arterial}^{25}$.

Com a intenção de avaliar a intensidade do efeito do suco de berinjela, comparamos o seu percentual de ação com outras drogas redutoras do colesterol. Em outro estudo experimental ${ }^{26}$, analisamos o efeito da pravastatina e da simvastatina em coelhos hipercolesterolêmicos, mas, em que as drogas foram administradas por um período de quatro semanas, em doses equivalentes de $10 \mathrm{e} 15 \mathrm{mg} /$ dia para a simvastatina e a pravastatina, respectivamente. O CT elevou-se em $2641 \%$ no $\mathrm{GH}$ e reduziu-se a valores percentuais de 58 e 57 para a simvastatina e a pravastatina, respectivamente. As LDL que elevaram-se a valores de $4796 \%$ no GH reduziram-se a percentuais de 64 e 63 para os grupos simvastatina e pravastatina, respectivamente. Os triglicérides diminuíram em $11 \%$ para a simvastatina e a pravastatina. Embora a comparação entre o tratamento com a berinjela e os inibidores da HMGCoA redutase difira no tempo de administração das substâncias, permite uma visão geral sobre suas intensidades de ação. Para níveis similares de elevação do colesterol plasmático, a simvastatina e a pravastina são muito mais efetivas em reduzir o CT e LDL plasmáticos. Entretanto, os TG diminuíram mais evidente- mente com o tratamento com a berinjela. Resta considerar a equivalência entre a dose utilizada nos animais tratados com berinjela, simvastatina e pravastatina. É possível que doses mais concentradas do suco de berinjela implementassem maior redução de CT. É importante assinalar que o suco de berinjela aumentou em $28 \%$ o relaxamento máximo dependente do endotélio e a pravastatina em $23 \%$ não havendo modificação significante para a simvastatina neste modelo de estudo. É possível que a ação mais evidente sobre o relaxamento dependente do endotélio observada nos animais tratados com a berinjela seja conseqüente não só da redução da hipercolesterolemia mas também de um efeito antioxidante sobre as LDL nativas, oxidadas e da parede arterial. Entretanto, não estudamos no experimento relatado, a eventual ação antioxidante da simvastatina e pravastatina.

A redução do peso corpóreo nos animais tratados com o suco de berinjela foi interpretado como conseqüente ao elevado teor de fibras encontrado na berinjela.

Os resultados deste estudo permitem concluir que o suco de berinjela, nas condições da experimentação, representa um tratamento alternativo e de baixo custo para as hipercolesterolemias, melhorando o perfil plasmático, o colesterol tecidual, a peroxidação lipídica das LDL nativas oxidadas e da parede arterial e provocando uma significativa proteção sobre o endotélio vascular.

\section{Referências}

1. Kannel WB, Casteli WP, Gordon TL - Cholesterol in the prediction of atherosclerotic disease. News perspectives based on the Framingham study. Ann Intern Med 1979; 90: 85-91.

2. Multiple Risk Factors Intervention Trial (MRFIT). Ann Intern Med 1985; 141: 1191.

3. Lipid Research Clinics Program. The Lipid Research Clinics Coronary Prevention Trial Research I and II. JAMA 1984; 251: 351-74.

4. Blankenhorn DH, Nessim AS, Johnson RL, Sanmarco ME, Azen SP, CashinHemphill L - Beneficial effects of colestipol-niacin therapy on coronary atherosclerosis and venous bypass graft. JAMA 1987; 257: 3233-40.

5. Buchwald H, Varco RL, Matts JP, Long JM, Fitch LL, Campbel GS - Effects of partial ileal bypass surgery on mortality and morbidity from coronary heart disease in patients with hypercholesterolemy. Report of the Program of the Surgical Control of Hyperlipidemias. N Engl J Med 1990; 323: 946-55.

6. Ornish D, Brorun SE, Schermitz LW, Billings JH, Armstrong WT, Ports TA - Can lifestyle changes reverse coronary heart disease? The lifestyle heart Trial. Lancet 1990; 336: 129-33.

7. Brown G, Albers JJ, Fisher LD et al - Regression of coronary artery disease as a result of intensive lipid lowering therapy in men high levels of apoliprotein B. N engl J Med 1990; 323: 1290-98.

8. Ribeiro Jorge PA, Ozaki MR, Eros A, Credidio L, Metze K-Effects of vitamin Eon endothelium dependent coronary flow hypercholesterolemic dogs. Atherosclerosis 1996; 126: 43-51.

9. Stewart-Lee AL, Forster LA, Nourouz-Zadheg J, Ferns GAA, Angaard EE- Vitamin E protects against impairement of endothelium-mediated relaxations in cholesterol-fed rabbits. Arteriosclerosis Thromb 1994; 14: 494-99.

10. Rimm EB, Stampfer MJ, Aschero A, Giovannucci E, Coldits GA, Willet WC-Vitamin E consuption and risk of coronary disease in men. NEngl J Med 1993; 328: 145-56.

11. Helrich K - Official Methods of Analysis of the Association of Official Analytical Chemists. AOAC. $15^{\text {th }}$ ed. Arlington, Virginia, 1990.

12. Naito HK, David JA - Laboratory considerations: determination of cholesterol, triglycerides, phospholipids and others lipids in blood and tissues. In: Laboratory and Research Methods in Biology and Medicine: Lipid Research Methodology. New York: Alan R. Liss, 1981: 1-76.

13. Havel RJ, Bragdon JH - The distribution and chemical composition of ultracen- trifugally separated lipoproteins in human serum. J Clin Invest 1955; 34: 134553.

14. Lowry OH, Rosebrough NJ, Farr AL, Randal AJ - Protein measurement with folin phenol reagent. J Biol Chem 1951; 193: 265-75.

15. Bueg JA, Aust SD - Microsomal Lipid Peroxidation. Methods of Enzimology. New York, 1978; 52: 302-10.

16. SAS (Statistical analysis System) SAS/TAT user's guide. Version $6,4^{\text {th }}$ ed, vol 1 , 1990.

17. Snedecor GW, Cochran WG-Statistical Methods. $7^{\text {th }}$ ed. Ames, Iowa: Iowa State University Press, 385-8.

18. Jayakody L, Seneratne M, Thomson A, Kapagoda T-Endothelium-dependent relaxation in experiment atherosclerosis in the rabbit. Circ Res 1987; 50: 251-64.

19. Nenseter Nenseter M, Gudmundesen O, Malterud K, Berg T, Drevon C-Effect of cholesterol feeding on the susceptibility of lipoproteins to oxidative modification. Biochimica Biophysica Acta 1994;1213: 207-14.

20. Kushwava R, Hazzard W - Catabolism of very low density lipoprotein in the rabbit effect of changing composition and pool size. Biochimica Biophysica Acta 1987; 528: 176-89.

21. Mitschek GH-Effect of solanum melongena on experimental atheromatosis. Histological studies on cholesterol-induced atheromatosis in rabbits in mean and long term test. Exp Pathol 1975; 10: 156-66.

22. Cruz J, Cruz HMM, Teixeira JEC et al - Tratamento das dislipidemias de hipertensos essenciais com berinjela (solanum melongena). Rev Soc Cardiol ESP 1997; 7(supl): 19.

23. Flick GJ, Burnette FS, Aung LH, Ory RL, Angelo AJ-Chemical composition and biochemical properties of mirlitons (sechium edue) and purple, green and white eggplants (solanum melongena). J Agric food Chem 1978; 26: 1000-5.

24. Kritchevsky D, Teppers AS, Story JA - Infuence of an eggplant (solanum melongena) preparation on cholesterol metabolism in rats. Exp Pathol 1975; 10: 180-3.

25. Ribeiro Jorge PA, Ozaki RM, Almeida E-Rapid reversal of endothelial dysfunction in hypercholesterolemic rabbits with simvastatin and pravastatin. Clin Exp Pharmacol Physiol (no prelo).

26. Ribeiro Jorge PA, Ozaki R, Metze K - Effects of pravastatin and simvastatin on endothelium dependent relaxation in hypercholesterolemic rabbits. Exp Tox Pathol 1994; 46: 465-9. 